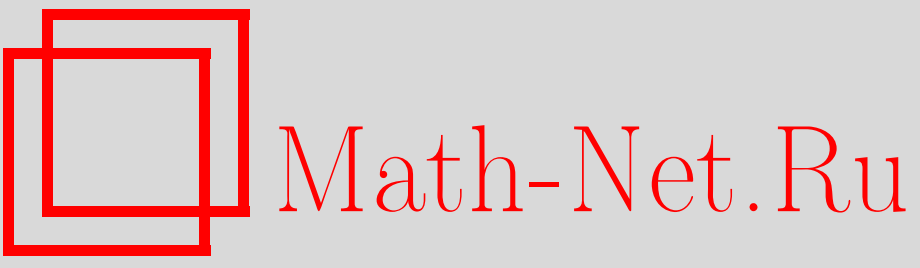

С. П. Новиков, Оператор Шрёдингера на графах и топология, УМН, 1997, том 52, выпуск $6,177-178$

DOI: https://doi.org/10.4213/rm910

Использование Общероссийского математического портала Math-Net.Ru подразумевает, что вы прочитали и согласны с пользовательским соглашением

http://www.mathnet.ru/rus/agreement

Параметры загрузки:

IP : 54.198 .187 .58

26 апреля 2023 г., 12:34:52 


\title{
ОПЕРАТОР ШРЁДИНГЕРА НА ГРАФАХ И ТОПОЛОГИЯ
}

\author{
С. П. Новиков
}

Определим оператор Шрёдингера для одномерного симплициального комплекса (графа) ${ }^{1} \Gamma$ без концов (т.е. в любой вершине сходится конечное число ребер, не меньшее двух), который действует на функции от вершин $T$ или ребер $R$ :

$$
(L \psi)_{T}=\sum_{T^{\prime}} b_{T: T^{\prime}} \psi_{T^{\prime}}
$$

для $L_{0}=\partial \partial^{*}, b_{T: T^{\prime}}=1, b_{T: T}=-m_{T}$,

$$
(L \psi)_{R}=\sum_{R^{\prime}} d_{R: R^{\prime}} \psi_{R^{\prime}}
$$

для $L_{0}=\partial^{*} \partial, d_{R: R^{\prime}}=1, d_{R: R}=-2$.

Здесь коэффициенты вещественны, симметричны и отличны от нуля толшко для ближайших соседей

$$
T^{\prime} \cup T=\partial R, \quad R \cap R^{\prime}=T .
$$

Коэффициенты $b_{T: T}=W_{T}$ и $d_{R: R}=W_{R}$ называются потенциалом, $m_{T}$ - это число ребер в вершине, $\partial$ - граничньй оператор.

ОПРеДЕЛЕНИЕ 1. Вронскианом пары решений $L \psi_{i}=\lambda \psi_{i}, i=1,2$, называется кососимметричное билинейное вьражение

$$
\begin{aligned}
& W_{\mathbf{R}}=b_{T: T^{\prime}}\left(\psi_{1 T} \psi_{2 T^{\prime}}-\psi_{2 T} \psi_{1 T^{\prime}}\right), \quad T^{\prime} \neq T, \\
& W_{\mathbf{R}}=\sum_{R^{\prime} \cap R=T} d_{R: R^{\prime}}\left(\psi_{1 R} \psi_{2 R^{\prime}}-\psi_{2 R} \psi_{1 R^{\prime}}\right), \quad R^{\prime} \neq R .
\end{aligned}
$$

Здесь $\mathbf{R}=\left(T T^{\prime}\right)$ это ориентированное ребро, $W_{T T^{\prime}}=-W_{T^{\prime} T}$.

Теорема 1. Вронскиан пары решений корректно определен как 1-цепь на Г, граница которой равна нулю: $\partial W=0$.

Доказательство вытекает в обоих случаях из рассмотрения величины $\left(L \psi_{1}\right) \psi_{2}-\left(L \psi_{2}\right) \psi_{1}$.

Рассмотрим граф $\Gamma$, имеющий $k$ "хвостов" $\left(z_{1}, \ldots, z_{k}\right), k \geqslant 1:$ хвосты $z_{j}$ являются полупрямыми с ребрами $R_{j n}, n \geqslant 1$, и вершинами $T_{j, n-1}, \partial R_{j n}=T_{j n}-T_{j, n-1}$. Пусть оператор Шрёдингера "финитен”, т.е. при $n>n_{0}$ и всех $j$ мы имеем: $L=L_{0}+2$ в обоих случаях.

Уравнение $L \psi=\lambda \psi$ имеет решения в $z_{j}$ при $n \rightarrow \infty$ вида $\psi_{j n}^{ \pm}=a_{ \pm}^{n}, a_{ \pm}=\frac{1}{2}\left(\lambda \pm \sqrt{\lambda^{2}-4}\right)$. Базис вещественных решений для $\lambda \in \mathbb{R}$ таков:

$$
C_{j n}=\left(a_{-} \psi_{j n}^{+}+a_{+} \psi_{j n}^{-}\right)\left(a_{+}+a_{-}\right)^{-1}, \quad S_{j n}=\left(\psi_{j n}^{+}-\psi_{j n}^{-}\right)\left(a_{+}-a_{-}\right)^{-1} .
$$

Эти решения определены только в хвостах. Введем “фазовое пространство” $\mathbb{R}^{2 k}$ с базисом $\left(C_{1}, S_{1}, \ldots, C_{k}, S_{k}\right)$ и кососкалярным произведением

$$
\left\langle C_{i}, C_{j}\right\rangle=\left\langle S_{i}, S_{j}\right\rangle=0, \quad\left\langle C_{i}, S_{j}\right\rangle=\delta_{i j}
$$

Оператор $L$ определяет подпространство $\Lambda_{\lambda}^{\infty} \subset \mathbb{R}^{2 k}$ векторов $\psi_{\infty} \in \Lambda_{\lambda}^{\infty}$, продолжающихся на весь граф $Г$ как решение

$$
L \psi=\lambda \psi, \quad \psi=\psi_{\infty}=\left(\psi_{1 \infty}, \ldots, \psi_{k \infty}\right), \quad \psi_{j \infty}=\alpha_{j} C_{j}+\beta_{j} S_{j}
$$

в хвосте $z_{j}$ для $n>n_{0}$.

\footnotetext{
${ }^{1}$ Обсуждение этой проблемы мы начали вместе с А. П. Веселовым.
} 
ТеОРема $2^{2}$. Подпространство $\Lambda_{\lambda}^{\infty}$ является лагранжевым, т.е. скалярное произведение на нем равно нулю.

Доказательство вытекает из теоремы 1. Действительно, для любой пары решений $\psi_{1}, \psi_{2}$ на $\Gamma$ мы имеем $W=\sum_{j=1}^{k} \varkappa_{j} z_{j}+$ (конечное), где $z_{j}-$ хвосты. Лишь разности $z_{i}-z_{j}$ продолжаются до циклов на $Г$ по модулю $\infty$. Поэтому мы имеем $W=\sum_{l \geqslant 2}^{k} \mu_{l}\left(z_{1}-z_{l}\right)+($ конечный цикл) $=\sum_{j=1}^{k} \varkappa_{j} z_{j}+$ (конечная цепь). Отсюда следует теорема

$$
\sum_{j=1}^{k} \varkappa_{j}=0=\left\langle\psi_{1 \infty}, \psi_{2 \infty}\right\rangle .
$$

Представим граф $\Gamma$ в виде $\Gamma=\Gamma^{\prime} \cup K_{1} \cup \cdots \cup K_{s}$, где $K_{l}-$ деревья, растущие из вершин ("гнезд") $T_{l} \in \Gamma^{\prime}$, причем $\Gamma^{\prime}-$ конечный граф̆ без концов ("основа"), $s \leqslant k$.

Tеорема 3. Размерность пространств решений $L \psi=\lambda \psi$ на $\Gamma$ всегда не меньше, чем $k$. Оно строго больше чем $k$ если и только если оператор Шрёдингера $L^{\prime}$, ограниченный на основу $\Gamma^{\prime}$, имеет “особое" собственное число $\lambda_{p}^{\prime}$, где $\psi_{p}^{\prime}\left(T_{l}\right)=0$ во всех гнездах $l=1, \ldots, s$. В этом и только әтом случае оператор, сопоставляющий решению $L \psi=\lambda \psi$ вектор $\psi_{\infty} \in \Lambda_{\lambda}^{\infty}$, имеет нетривиальное ядро.

Спектр оператора $L$ в гилбертовом пространстве $\mathscr{L}_{2}^{\varepsilon}(\Gamma)=H_{\varepsilon}, \varepsilon=0,1$ (вершины и ребра) делится на непрерьвную часть $|\lambda| \leqslant 2$ (зона рассеяния) и дискретньй спектр в зонах $\lambda<-2$, $\lambda>2$. Точки дискретного спектра внутри зоны $|\lambda| \leqslant 2$ возможны лишь локализованные на основе $\Gamma^{\prime}$, где $\lambda=\lambda^{\prime}$ - "особое" собственное число. Алгебраические свойства зоны рассеяния ("унитарность") целиком определяются теоремой 2 . При $|\lambda|>2$ мы имеем $a_{+} a_{-}=1, a_{ \pm} \in \mathbb{R}$. Рассмотрим лагранжеву плоскость $\Lambda_{\lambda}^{-} \subset \mathbb{R}^{2 k}$ с убьвающим на бесконечности базисом $\Psi_{j}^{-}$, $j=1, \ldots, k$.

ПрЕДЛОЖЕНИЕ 1. Точка $\lambda \in \mathbb{R},|\lambda|>2$, является точкой дискретного спектра для L в $H_{\varepsilon}(\varepsilon=0,1)$, если $\Lambda_{\lambda}^{\infty} \cap \Lambda_{\lambda}^{-}-$непусто или $\lambda=\lambda_{p}^{\prime}$ - особое собственное число на основе. Индексы Морса кривых $\Lambda_{\lambda}^{\infty}$ в лагранжевом грассмане совпадают с алгебраическими числами собственных значений для $\lambda \in[2, \infty]$ u $\lambda \in[-\infty,-2]$. Дискретный спектр заведомо имеется если: $\max _{T}\left(\sum b_{T: T^{\prime}}^{2}+W_{T}^{2}\right)>4, \max _{R}\left(\sum d_{R: R^{\prime}}^{2}+W_{R}^{2}\right)>4$ или $\max _{q}\left|\lambda_{q}^{\prime}\right|>4$ для спектра основы $\Gamma^{\prime}$ (в том числе неособого).

\section{СПИСОК ЛИТЕРАТУРЫ}

[1] Новиков С. П., Дынников И. А. // УМН. 1997. Т. 52. № 5. С. 175-234. [2] Новиков С. П. // Изв. АН СССР. Сер. матем. 1970. Т. 34. № 2. С. 253-288; № 3. С. 475-500.

University of Maryland at College Park

E-mail: novikov@ipst.umd.edu

Принято редколлегией 25.11.1997

\footnotetext{
${ }^{2}$ В 1971 г. И. М. Гелшфанд сообщил автору интересную идею о связи самосопряженных расширений симметрических операторов с лагранжевьми плоскостями. Это было реакцией Гельфанда на работу автора [2], построенную на связи между гамильтоновым формализмом и дифференциальной топологией.
} 